\title{
Observation of Ionospheric Gravity Waves Introduced by Thunderstorms in Low Latitudes China by GNSS
}

\author{
Tong Liu ${ }^{1}{ }^{\mathbb{D}}$, Zhibin $\mathrm{Yu}^{1}{ }^{1} * \mathbb{D}$, , Zonghua Ding ${ }^{2}$, Wenfeng $\mathrm{Nie}^{3}$ and Guochang $\mathrm{Xu}^{1}$ \\ 1 Institute of Space Science and Applied Technology, Harbin Institute of Technology, Shenzhen 518055, China; \\ 19b958024@stu.hit.edu.cn (T.L.); xuguochang@hit.edu.cn (G.X.) \\ 2 National Key Laboratory of Electromagnetic Environment, Qingdao 266107, China; dingzh@crirp.ac.cn \\ 3 Institute of Space Science, Shandong University, Weihai 264209, China; wenfengnie@sdu.edu.cn \\ * Correspondence: yuzb@hit.edu.cn; Tel.: +86-755-2661-1417
}

check for updates

Citation: Liu, T.; Yu, Z.; Ding, Z.; Nie, W.; Xu, G. Observation of Ionospheric Gravity Waves Introduced by Thunderstorms in Low Latitudes China by GNSS. Remote Sens. 2021, 13, 4131. https://doi.org/10.3390/ rs13204131

Academic Editor: Mattia Crespi

Received: 29 August 2021

Accepted: 13 October 2021

Published: 15 October 2021

Publisher's Note: MDPI stays neutral with regard to jurisdictional claims in published maps and institutional affiliations.

Copyright: (c) 2021 by the authors. Licensee MDPI, Basel, Switzerland. This article is an open access article distributed under the terms and conditions of the Creative Commons Attribution (CC BY) license (https:// creativecommons.org/licenses/by/ $4.0 /)$.

\begin{abstract}
The disturbances of the ionosphere caused by thunderstorms or lightning events in the troposphere have an impact on global navigation satellite system (GNSS) signals. Gravity waves (GWs) triggered by thunderstorms are one of the main factors that drive short-period Travelling Ionospheric Disturbances (TIDs). At mid-latitudes, ionospheric GWs can be detected by GNSS signals. However, at low latitudes, the multi-variability of the ionosphere leads to difficulties in identifying GWs induced by thunderstorms through GNSS data. Though disturbances of the ionosphere during low-latitude thunderstorms have been investigated, the explicit GW observation by GNSS and its propagation pattern are still unclear. In this paper, GWs with periods from 6 to $20 \mathrm{~min}$ are extracted from band-pass filtered GNSS carrier phase observations without cycle-slips, and 0.2-0.8 Total Electron Content Unit (TECU) magnitude perturbations are observed when the trajectories of ionospheric pierce points fall into the perturbed region. The propagation speed of $102.6-141.3 \mathrm{~m} / \mathrm{s}$ and the direction of the propagation indicate that the GWs are propagating upward from a certain thunderstorm at lower atmosphere. The composite results of disturbance magnitude, period, and propagation velocity indicate that GWs initiated by thunderstorms and propagated from the troposphere to the ionosphere are observed by GNSS for the first time in the low-latitude region.
\end{abstract}

Keywords: thunderstorm; ionospheric; gravity wave; traveling ionospheric disturbance; GNSS remote sensing

\section{Introduction}

The solar activities have effects on the ionospheric electron content from the top, which can be retrieved by the global navigation satellite system (GNSS) [1,2]. In recent years, perturbations from the underlying atmosphere have been found to be another important factor in ionospheric variability [3]. In addition to earthquakes [4] and typhoons [5], which have been explored extensively, lightning or thunderstorms can drive significant ionospheric perturbations. Ionospheric perturbations induced by thunderstorms can be propagated by gravity waves (GWs) as carriers, which have been reported both in midlatitudes and low-latitudes [6,7].

In mid-latitudes, the ionospheric Total Electron Contents (TECs) obtained by GNSS measurements with and without thunderstorms were compared [7]. Large ionospheric disturbances induced by thunderstorms were reported, and the maximum variation was about 1.4 Total Electron Content Units (TECUs) $\left(1 \mathrm{TECU}=10^{16} \mathrm{e} / \mathrm{m}^{2}\right)$. During the periods without thunderstorms, no detectable GW was found. When the Ionospheric Pierce Points (IPPs) of the GNSS signal path were above the thunderstorms, high-frequency (period about $4 \mathrm{~min}$ ) perturbations were present [8]. It was deemed that the source of the disturbance could be located from the infrasound observations from different GNSS stations. The positioning results showed that the disturbance sources were in the regions of thunderstorm monoliths or strong convection, suggesting that the infrasound disturbances were 
triggered by thunderstorms. The statistical study from North America further elucidated the correlation between thunderstorms and ionospheric GWs at mid-latitudes [6].

Compared with the obvious observation of the ionospheric GWs induced by thunderstorms at mid-latitudes, GW observation through GNSS data is difficult in low latitudes, likely due to the low-latitudes complex ionospheric dynamical processes. The statistical correlation study [9] suggested that thunderstorms could drive the unstable post-sunset ionosphere to a state with significant spatial irregularities. Moreover, when the ionospheric spatial irregularities do not dominate, thunderstorm-induced acoustic and GWs could be observable in the low-latitude ionosphere. The band-pass filters could be used to extract ionospheric GWs at specific periods. A positive correlation between the amplitudes of band-pass filtered GW and thunderstorm activities was found [10]. Besides the wave-like ionospheric disturbances during thunderstorms, a TEC variations analysis [11] showed that ionospheric anomalies could reach up to $70 \mathrm{TECU} / \mathrm{min}$. This magnitude was significantly larger than the disturbances in Brazil [9] and West Africa [12]. Further, wavelet analysis was used to analyze the possible causes of ionospheric disturbances under lightning. It was shown that a combination of plasma bubbles and GWs could be responsible for the 10-100 min disturbance periodicity [11]. The ionospheric disturbances brought about by thunderstorms during the daytime were analyzed in West Africa [12]. It was pointed out that visible GWs by GNSS were correlated with thunderstorms and propagated in a specific direction. However, in comparison with the clearly observable GWs caused by typhoons [13-15], the direct observations of GWs introduced by thunderstorms are unclear through GNSS in low latitudes. The ionospheric GWs in the low-latitude/equatorial region induced by thunderstorms need to be further investigated.

Considering that GWs propagate in the atmosphere before reaching the ionosphere, the properties of GWs should be explored from the perspective of atmospheric medium propagation theory [16]. This study mainly focuses on 1, determining the observability and magnitude of GWs induced by lightning at low latitudes; 2, investigating the period, propagation direction, distance, and speed of GWs in the ionosphere; and 3, the relationship between the period characteristics of GWs and the propagation distance considering the atmospheric conditions.

The structure of the article is organized as follows: in Section 2, the data used in this paper are introduced; in Section 3, the methods of data processing are described; in Section 4, the observation results and characteristics of GWs under thunderstorms/lightning are analyzed; in Section 5, the results are discussed in comparison with existing studies; and Section 6 contains the Summary.

\section{Data}

\subsection{GNSS Raw Data}

GPS dual-frequency carrier phase in Receiver Independent Exchange (RINEX) format for 2-5 April 2019 for stations at low latitudes $\left(105-120^{\circ} \mathrm{E} 15-30^{\circ} \mathrm{N}\right)$ in China from National Earthquake Data Center (https:/ / data.earthquake.cn/ (accessed on 12 October 2020)) and all stations in the Hong Kong Geodetic Network (https:/ / www.geodetic.gov.hk/ (accessed on 5 April 2021)) were used. The sampling rate of the data was $30 \mathrm{~s}$.

\subsection{Lightning Data}

The data included the lightning occurrences number, magnitude, and location, which were obtained from the lightning meter 1-min event product of the lighting mapping imager (LMI) of FY-4 satellite (http:/ / data.cma.cn/data/cdcdetail/dataCode/SK.0983.001.html (accessed on 16 July 2021)) and the ground-based observations from the Institute of Electrical Engineering Chinese Academy of Sciences (http:/ / www.iee.ac.cn/ (accessed on 13 April 2021)). 


\subsection{Kp and Dst Index}

The Kp-index is an index used by individual geomagnetic stations to describe the intensity of geomagnetic disturbances every $3 \mathrm{~h}$ of the day. The Dst index was measured every hour, mainly to measure the variation in the horizontal component of the geomagnetic field. The Kp and Dst indexes are available from: http://wdc.kugi.kyoto-u.ac.jp/index.html (accessed on 1 June 2021).

\section{Methods}

\subsection{Extraction of Ionospheric Disturbances}

First, the L1 and L2 carrier phase data are used to do geometry-free combinations. For low latitudes, especially in areas where thunderstorms are frequent, the handling of cycle slips is tricky. The presence of undetected cycle slips may lead to great TECU errors in the results. For ionospheric disturbances extraction, the calculation of the absolute TECU values is not necessary. The cycle slips can be treated as biases using the time difference method $[17,18]$. The ambiguity is similarly eliminated during the time differencing process. By the method of using only the carrier phase to obtain the ionospheric perturbation, the accuracy of the total electron content on the signal path can reach 0.03 TECU [13]. The detailed derivation of formulas can be found in studies of Savastano et al. [17] and Jian et al. [13].

\subsection{Extraction of Ionospheric Gravity Waves}

GWs associated with thunderstorms typically have a short period of 6-20 $\mathrm{min}[9,10]$. For the extraction of such disturbances with known periods, a band-pass filter can be used $[6,9]$. The passband of the filter is $0.8-2.8 \mathrm{mHz}$. Due to the presence of apparent dispersive shifts in the unidirectional bandpass filter, a bidirectional bandpass filter needs to be utilized. The bandpass filter used in this study is a bidirectional Butterworth filter [19].

\subsection{GW Period with Propagation Distance}

The periodic term of GWs is integrated into the atmospheric dynamical equations. In the case of a normal wind field (wind speed below a first-order typhoon) and without considering the Coriolis force, the dispersion equation of high-frequency waves is $[16,20,21]$ :

$$
\hat{\omega}^{2}=\frac{N^{2} k_{\mathrm{h}}^{2}}{k_{\mathrm{h}}^{2}+m^{2}}=N^{2} \cos ^{2} \alpha
$$

where $\hat{\omega}$ denotes the GW intrinsic frequency and $N$ denotes the Brunt-Vaisala frequency. Horizontal wave number $k_{\mathrm{h}}=2 \pi / \lambda_{\mathrm{h}}, \lambda_{\mathrm{h}}$ is the horizontal wavelength; vertical wave number $m=2 \pi / \lambda_{z}, \alpha$ is the angle between the horizontal propagation direction of the GW and the vertical direction. Denoting the GW period by $\tau$, from $\hat{\omega}=2 \pi / \tau$ [16]:

$$
\tau=\frac{2 \pi}{N} \sec \alpha=\frac{2 \pi}{N} \sqrt{1+\frac{R^{2}}{\Delta z^{2}}}
$$

$\Delta z$ denotes the vertical distance from the height of the GW source to the thin ionospheric shell. The default ionospheric thin shell height of $300 \mathrm{~km}$ is set in the calculation. $R$ denotes the horizontal distance from the observed GW to the wave source. Equation (2) expresses the period of the GW as a function of the horizontal propagation distance. In the equation, the Brunt-Vaisala frequency is taken as $N=2 \pi / 5 \mathrm{~min}^{-1}$ [16]. Therefore, when the period of the GW is observed, the horizontal propagation distance can be determined when the ionospheric height is known.

\section{Results}

\subsection{Extraction of GWs from Time Series}

First, the consistency of thunderstorm occurrence and GW observations is analyzed in terms of time. 
The satellites over the low latitudes of China at 12-20 h are pseudo random noise code (PRN) 2, 3, 5, 6, 9, 17, 19, 28, and 30. Using a polynomial fit, after removing the trend of daily variation, all satellites still have cyclical disturbances in hourly units. The results of the perturbation before removing the trend term with long periods are shown in the left panel of Figure 1.
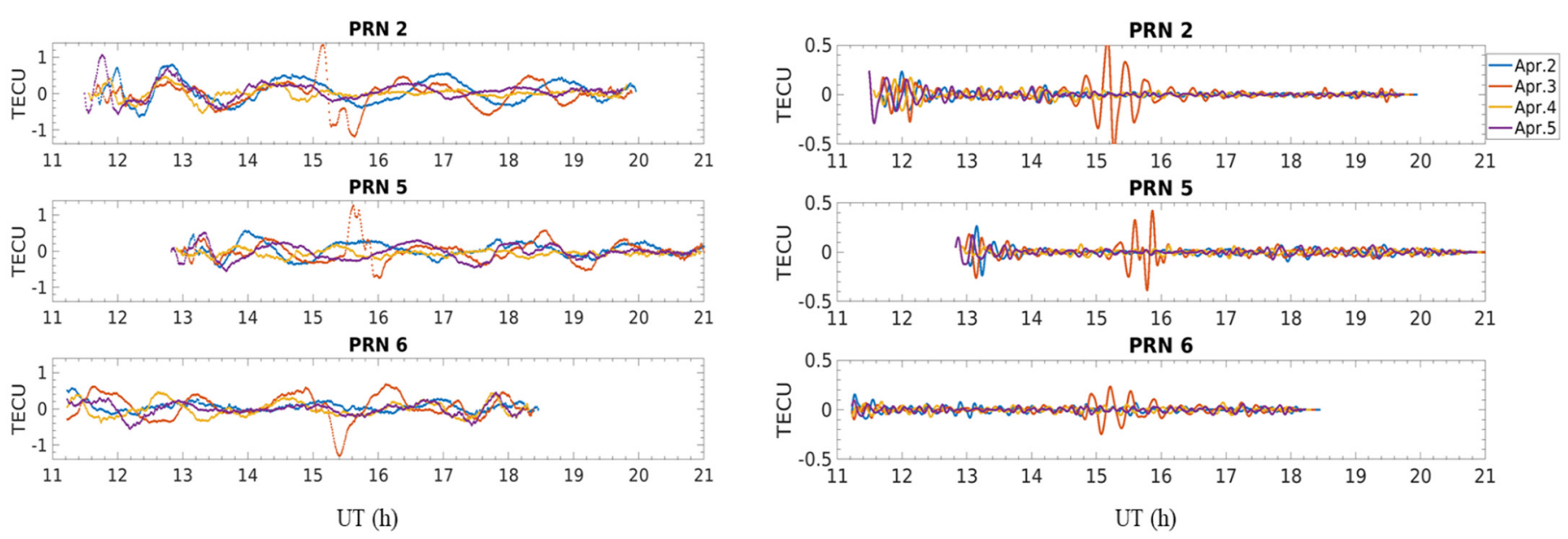

Figure 1. Observations of PRN 2, 5, and 6 at the HKWS station from 11:00 to 21:00 on 2-5 April 2019. The left panel indicates the disturbance and the right panel indicates the ionospheric GW. The three subplots indicate PRN 2, 5, and 6. The colors of the lines represent different dates: blue for 2 April, red for 3 April, yellow for 4 April, and purple for 5 April.

After processing with band-pass filters, only PRN 2, 5, and 6 have distinct disturbances with a period of 6-20 min at around 15-16 h, which are in the range of the period of lightning-induced GWs $[6,9,10]$, as the right panel shows. Figure 1 only shows the results of the HKWS station; the results of most stations in Hong Kong, China are consistent with HKWS.

To explore whether the observations are triggered by a thunderstorm, the number of lightning events at 12-16 h from 2-5 April are used as a reference, as shown in Figure 2.
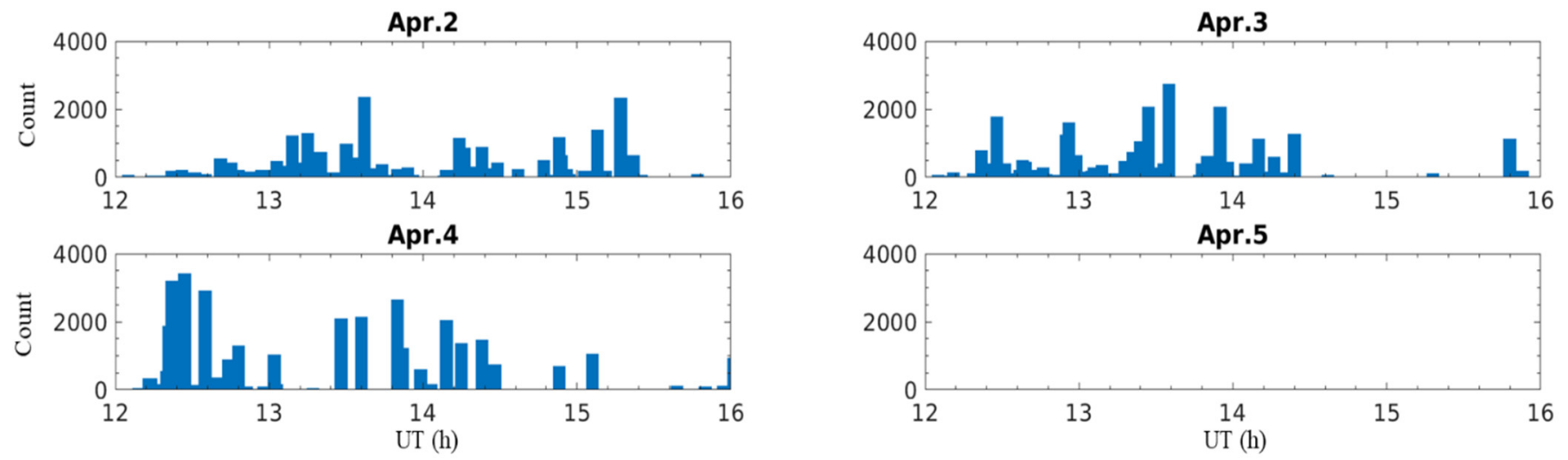

Figure 2. Lightning frequency and intensity obtained from the FY-4 satellite. The horizontal coordinate indicates the UTC in hours; the vertical bar indicates the sum of lightning events.

The GW observed at $15-16 \mathrm{~h}$, considering the $1-2 \mathrm{~h}$ delay $[10,11,22]$, is probably generated at 13-14 $\mathrm{h}$ and coincides with the lightning peak time of 3 April 2019. The lighting event from LMI is also recorded on April 2 and April 4; however, no satellites show observable GWs. No lightning is recorded on April 5. The results in Figures 1 and 2 tentatively show that, although there is no inevitable causal relationship between GW generation by thunderstorms and its GNSS observation, but some TECU variations can be associated with the thunderstorms. 


\subsection{Location of Thunderstorms and GWs}

To further investigate the spatial location relationship between ionospheric GWs and lightning, the map of lightning position using LMI data is shown in Figure 3. The GWs observed on April 3 are located directly north of Hong Kong and Shenzhen, as shown in the upper right subplot of Figure 3. GWs are not observed in the same area on other days. Around Vietnam and Laos (about $105^{\circ} \mathrm{E}, 18^{\circ} \mathrm{N}$ ), located on the southwest of China, there are longer period waves in days with and without thunderstorms.

Apr. 2

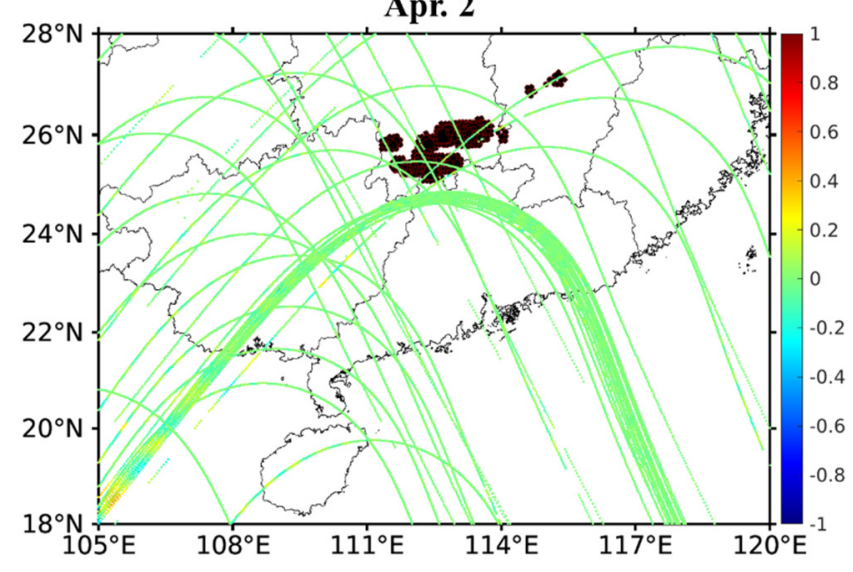

Apr. 4

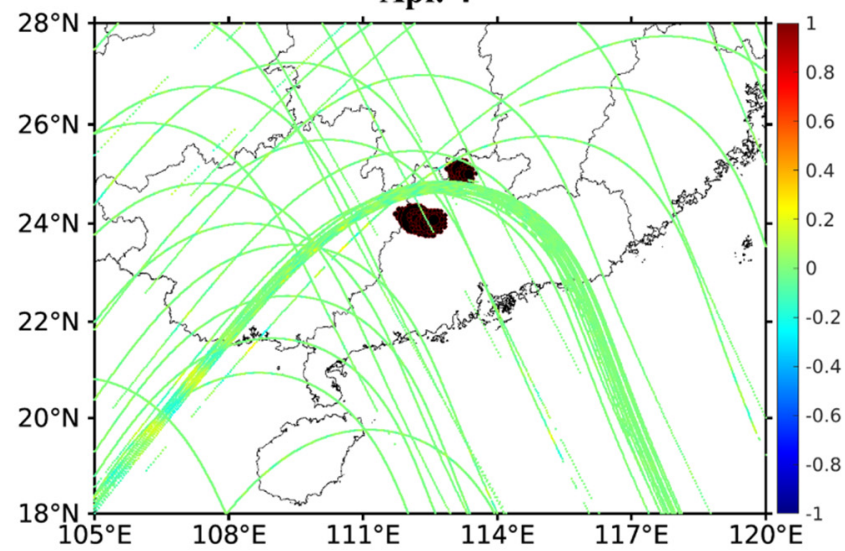

Apr. 3

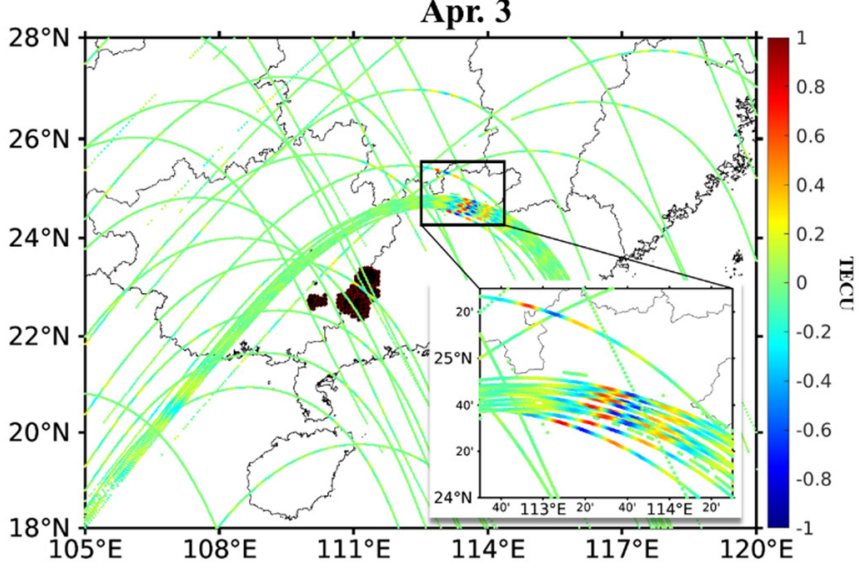

Apr. 5

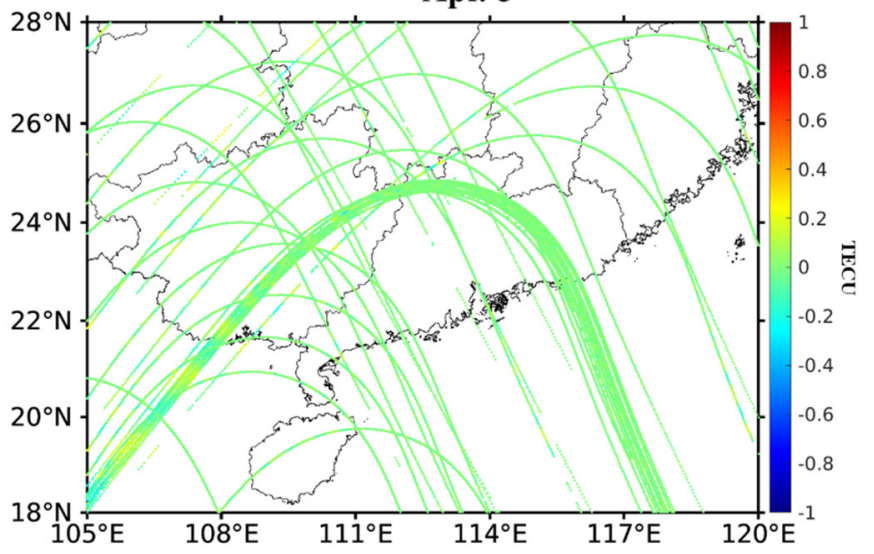

Figure 3. GW observations extracted from stations at low latitudes in China on 2-5 April 2019. The universal time is 10-16 h, i.e., 18-24 h local time. Different curves indicate the ionospheric penetration point trajectories of PRN2 observed by different base stations; the color axis indicates the amplitude of ionospheric GWs in TECU. Black patches show thunderstorm areas.

As shown in Figure 3, the April 3 thunderstorm is located near the location of the GWs: the thunderstorm location is the western part of Guangdong Province (about $113^{\circ} \mathrm{E}$, $23^{\circ} \mathrm{N}$ ), and the ionospheric GW location is about $114^{\circ} \mathrm{E}, 24^{\circ} \mathrm{N}$. There are no thunderstorms in other areas during that period. This suggests that the GWs observation on April 3 are most likely caused by nearby thunderstorms.

Even if thunderstorms existed on April 2 and 4, GWs similar to those on April 3 are not detected. This suggests that although the observed GWs may be triggered by thunderstorms, not all thunderstorms can trigger GWs and then be captured by GNSS observations. It is possible that GWs would propagate in a specific direction and not necessarily intersect with the IPP trajectory. The perturbation would only be captured if the GWs could cross the GNSS signal path.

On April 2 and April 4, the disturbances observed by PRN 2 are located on the southwest of China $\left(\sim 105^{\circ} \mathrm{E}, 18^{\circ} \mathrm{N}\right)$, but there are no local thunderstorms. The only thunderstorm 
area is located north of Guangdong $\left(\sim 112^{\circ} \mathrm{E}, 26^{\circ} \mathrm{N}\right)$. The thunderstorm region is far from the disturbance region, which suggests that there should be no correlation between the two.

In addition, comparing the results of April 2, 4, and 5, it can be found that there are slight GWs on the southwest of China $\left(\sim 105^{\circ} \mathrm{E}, 18^{\circ} \mathrm{N}\right)$ regardless of the presence of thunderstorms. This further verifies that the presence of waves in the region is not caused by thunderstorms in and around Guangdong.

\subsection{GW Propagation Direction}

The thunderstorm area is located at $111^{\circ} \mathrm{E}, 23^{\circ} \mathrm{N}$ and the $\mathrm{GW}$ observation area is located at $114^{\circ} \mathrm{E}, 24^{\circ} \mathrm{N}$. If the GW is triggered by a thunderstorm, then the direction of propagation of the GW is north of east. On the condition that the stations are in columns, the GW direction of propagation can be observed visibly from the time-series perturbation maps of multiple stations simultaneously or in sequence [23]. However, the stations in our study are dispersed. Thus, the relative position of all the stations needs to be ordered. As shown in Figure 4, in general, these three satellites PRN 2, 5, and 6 move from southwest to northeast. Therefore, all stations are ordered according to the southwest-northeast direction of their positions, as shown in Figure 5.
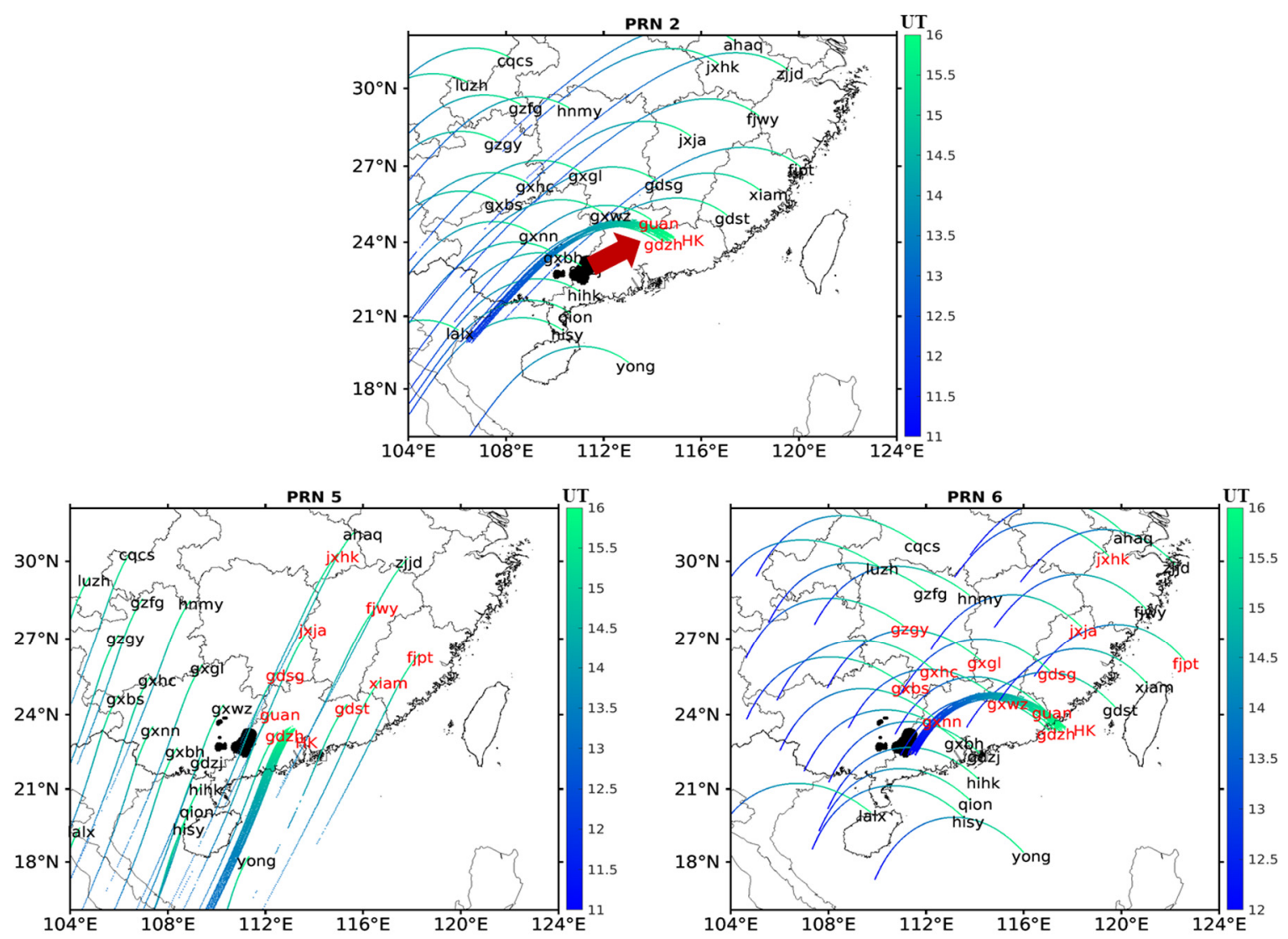

Figure 4. Trajectories of the satellite IPP of PRN 2, 5, and 6 from $14 \mathrm{~h}$ to $17 \mathrm{~h}$ on 3 April 2019. The color axis represents universal time. The black patches are thunderstorm areas. Hong Kong's dense stations are represented by 'HK'. The names of disturbed stations before and around $16 \mathrm{~h}$ are marked in red. 

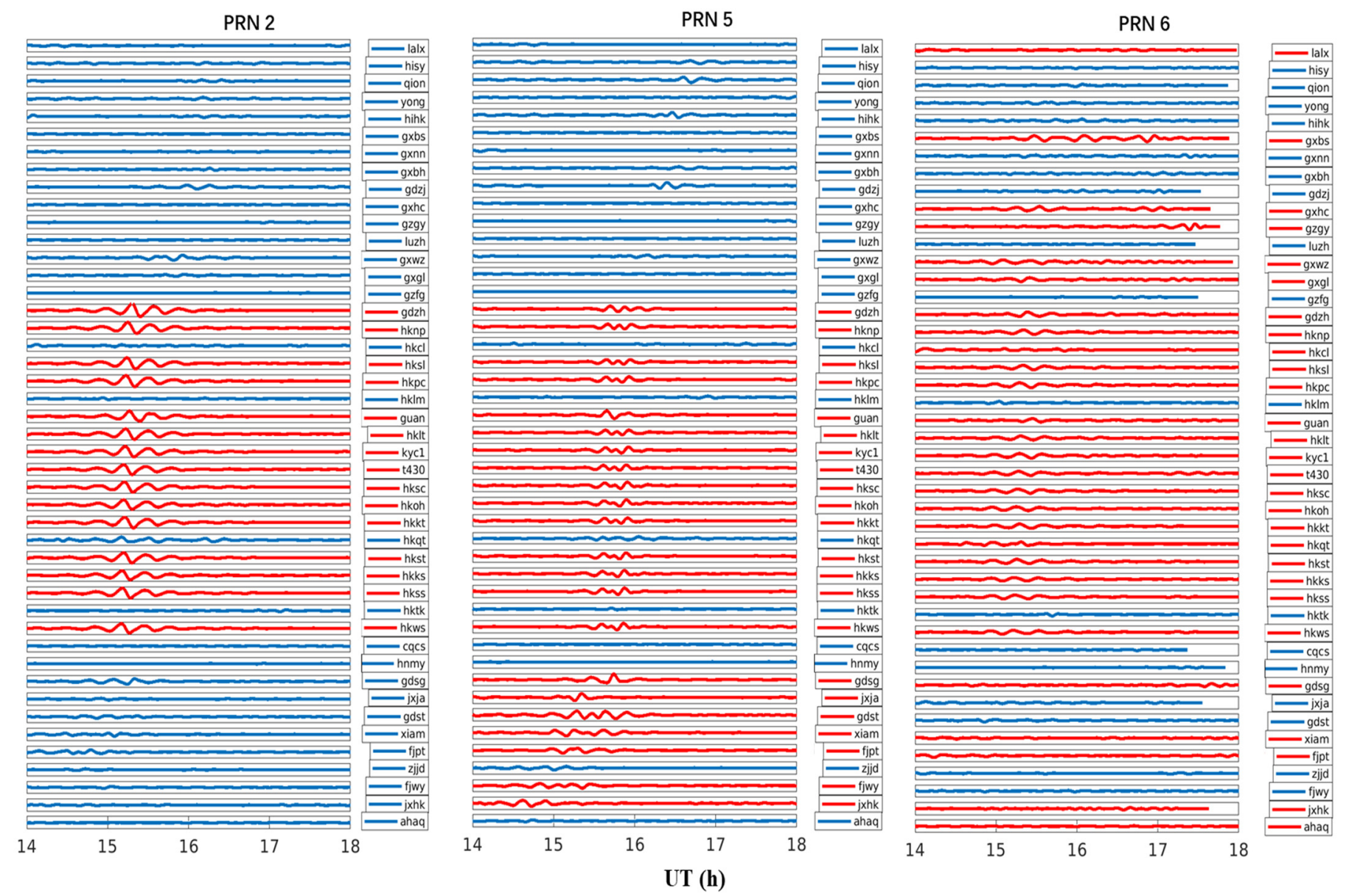

Figure 5. TEC time series for satellites 2, 5, and 6 on 3 April 2019. The legend shows the station names. The y-axis limits from $[-0.8,0.8]$ TECU for each column. The names of disturbed stations before and around $16 \mathrm{~h}$ are marked in red.

In the first subplot of Figure 4, The Hong Kong and Guangdong stations (GUAN and GDZH) of PRN 2 crossed the GW region at around $16 \mathrm{~h}$, while the IPPs of other stations did not cross the region. Therefore, as shown in the left panel of Figure 5, only the stations in and around Hong Kong are disturbed. The direction of the IPP motion of PRN 2 is similar to the direction of GW propagation, pointing from the black area to the area marked by the red station name but at different speeds. Therefore, theoretically, the two motions cross only once, i.e., the fluctuations can be observed only at a particular time.

In the second subplot of Figure 4, during the motion of the PRN 5 satellite, the IPP in the northeast is more likely to fall into the range of the GW first. Therefore, before 15:30, the stations in Jiangxi, Fujian, and Zhejiang (JXHK, FJWY, and JXJA) observe the disturbances first. With time, the stations in and around Hong Kong fall into the GW region only; finally, the IPPs of GDZJ in Guangdong and HIHK, QION in Hainan enter the GW range.

In the last subplot of Figure 4, during the motion of PRN 6, the IPP trajectories of the stations located in Guangdong and Guangxi first move northeastward and crossed over the thunderstorm; after that, they move southeastward and fell into the GW region. This results in the GWs of PRN 6 from different stations being observed at different times, especially the stations in Guangxi, where the thunderstorms originate.

The perturbation magnitude of PRN 2 is the largest ( \pm 0.8 TECU) among the observations from the three satellites. Observations from most of the dense stations in Hong Kong show the consistency of this magnitude. Figure 5 shows that the GW observation of PRN 2 is just after 15:00. At this time, the station of PRN 5 leaning to the east is also in the GW range and has a large disturbance. When the Hong Kong stations of PRN 5 fall into the 
GW region at 15:30, the amplitude has decayed ( $\pm 0.5 \mathrm{TECU})$. This indicates that the time when PRN 2 crosses the disturbance region is closer to the time when the GW is largest.

The initial disturbance times of the Hong Kong and additional stations of PRN 2 and PRN 6 are more consistent. However, spatially, the IPPs of PRN 6 are located at $117^{\circ} \mathrm{E}$, $24^{\circ} \mathrm{N}$, further away from the thunderstorm region than the $113^{\circ} \mathrm{E}, 24^{\circ} \mathrm{N}$ of PRN 2 . The farther position of PRN 6 leads to an attenuation of the magnitude ( \pm 0.4 TECU).

\subsection{Properties of the Gravity Wave Period}

The propagation period is a primary property of GWs. Figures 6 and 7 show the period characteristics obtained using the $S$ transform [14,24]. The occasion of perturbation observed by PRN 2 is around 15:00 for most stations, which is consistent with the left panel of Figure 5. The frequency of the disturbances is $1-2 \mathrm{mHz}$, corresponding to a period of 8.3-16.7 min, which is exactly the range of GWs induced by thunderstorms [6].
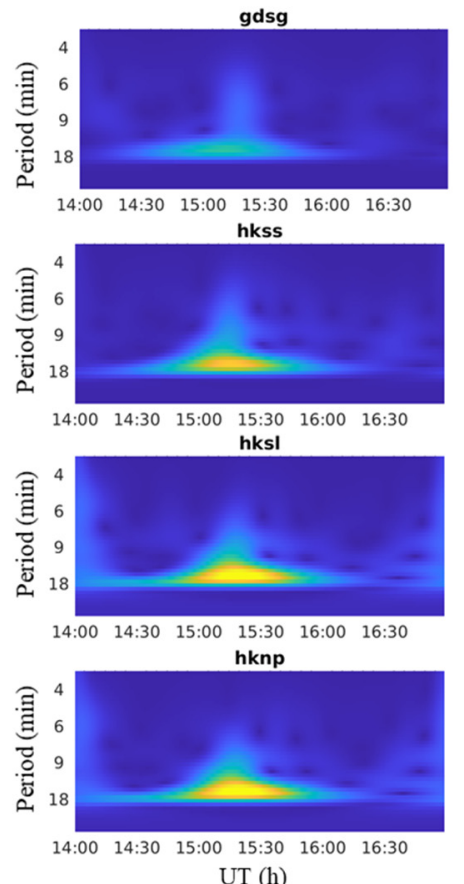

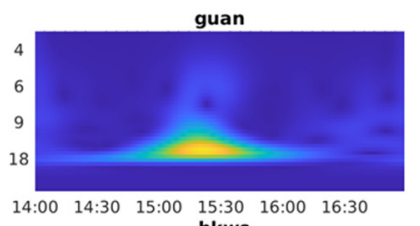

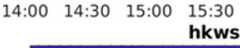
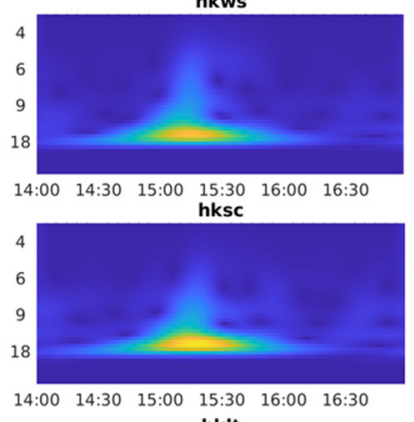

hklt

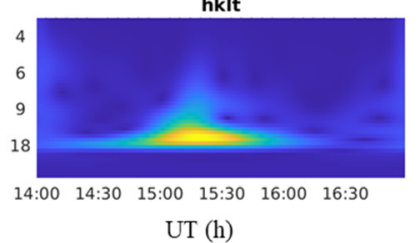

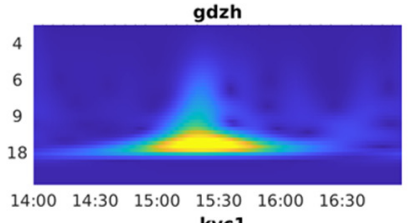

$\begin{array}{llll}14: 00 & 14: 30 & 15: 00 & 15: 30 \\ & & & \text { kyc }\end{array}$
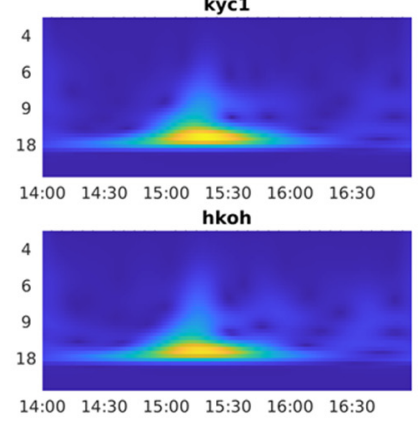

hkkt

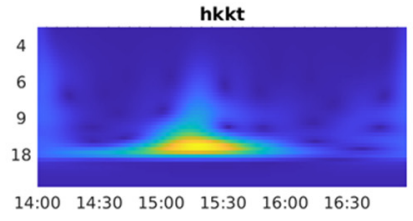

UT (h)
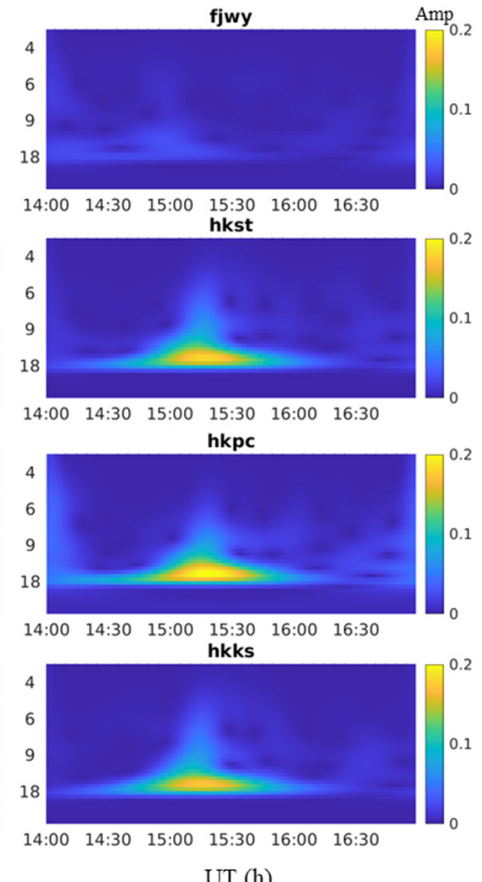

Figure 6. Wavelet analysis of PRN 2 from multiple station observations on 3 April 2019 to explore GW perturbation periodicities. The horizontal coordinate is universal time; the vertical coordinate is period; and the color axis is the perturbation magnitude. The characteristics of the period of PRN 6 are similar to PRN 2.

The majority of stations with large magnitudes of disturbances are those in Hong Kong. The stations named GDZH and GUAN are located in south Guangdong, adjacent to Hong Kong, so their disturbances are similar. The GDSG station is located in northern Guangdong. GDSG station observes small-magnitude GWs with similar periods to the Hong Kong station at about the same time.

The disturbance can be obtained from the GNSS signal only when the IPP trajectory intersects with the GW propagation trajectory. Geometrically, there is only one possible encounter between two objects moving in the same direction with different speeds. The eastward direction of the IPP motion of PRN 2 and PRN 6 is similar to the direction of GW propagation but at different speeds. Therefore, theoretically, these two motions only intersect once, i.e., the disturbance can be observed only at one particular time. 

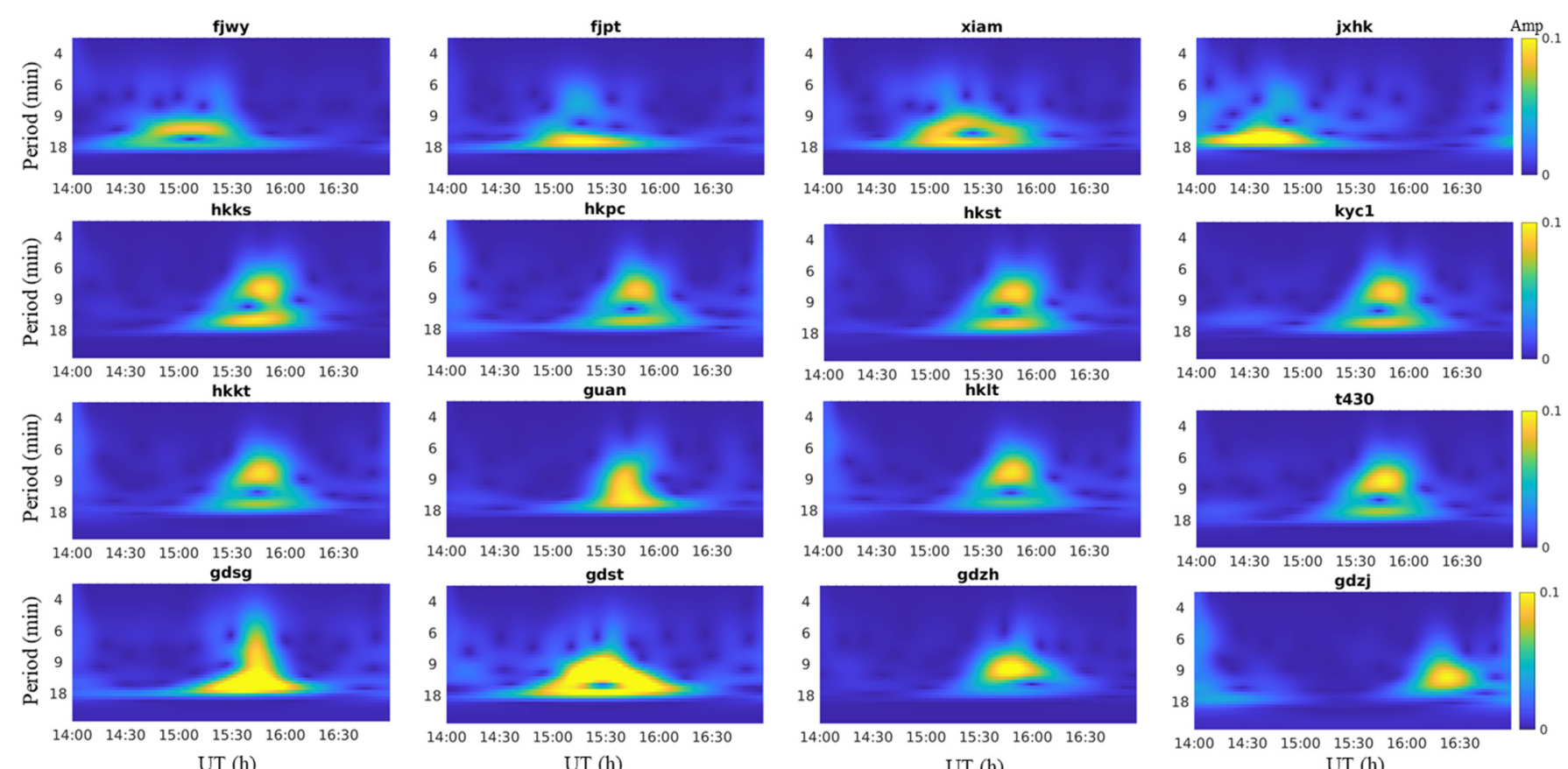

Figure 7. Wavelet analysis of PRN 5 from multiple station observations on 3 April 2019 to explore GW perturbation periodicities.

The multiple IPP trajectories of PRN 5 intersects the GW propagation direction at multiple locations; thus, the disturbance is more likely to be observed multiple times. The observation of PRN 5 is shown in Figure 7. The IPPs of five stations (JXHK, FJPT, FJWY, XIAM, and JXJA) located in Jiangxi and Fujian, which are at the easternmost side, first sense the GW before 15:30. After that, the IPPs deviate from the GW-induced disturbance area as the satellite moved. Then, between 15:30 and 16:30, the IPPs of stations around Guangdong and Hong Kong fall into the GW region. Although these stations observe GWs at different times, the observed frequencies are all in the range of about $1-3 \mathrm{mHz}$ (5.6-16.7 $\mathrm{min})$.

As shown in Figure 7, two perturbation periods of about 6-9 min and 18 min exist for most of the stations. This is due to the fact that the obtained observations are the total number of electrons on the line between the GNSS station and the satellite. The signal passes through the top and bottom of the ionosphere successively. It is possible that the GW periods of 6-9 $\mathrm{min}$ are at the bottom of the ionosphere and GW periods of around $18 \mathrm{~min}$ at the top. The continuous excited GWs lead to the simultaneous presence of perturbations of different periods in the ionosphere.

The GWs with periods of 6-9 min are only significantly observed by PRN 5 but not PRN2. One possible reason is that the main propagation direction of the GW with a period of 6-9 min is east-south, intersecting only the trajectory of PRN 5.

\subsection{The Relationship between GW Propagation Distance and Period}

To further determine the periodic characteristics, the theory that the period of GWs in the atmosphere varies with the propagation distance is introduced. Equation (2) indicates that different ionospheric heights produce inconsistencies in the theoretical values of the wave period. As shown in Figure 8, the four colored solid lines indicate the theoretical periods of GWs versus horizontal propagation distance for different ionospheric thinshell assumptions. In the legend, $60 \mathrm{~km}$ and $90 \mathrm{~km}$ represent the bottom and top of the ionospheric layer $\mathrm{D}$, and $150 \mathrm{~km}$ and $450 \mathrm{~km}$ represent the bottom and top of layer F. Layer $\mathrm{E}$ is between layers $\mathrm{D}$ and $\mathrm{F}$. The wave source location is set to $111^{\circ} \mathrm{E}, 23^{\circ} \mathrm{N}$, which is the central region of the thunderstorm during 13:00-15:00 on April 3. The distance marked on 
the horizontal axis is the Euclidean distance between the location of the IPP and the center of the thunderstorm in the WGS-84 coordinate system.
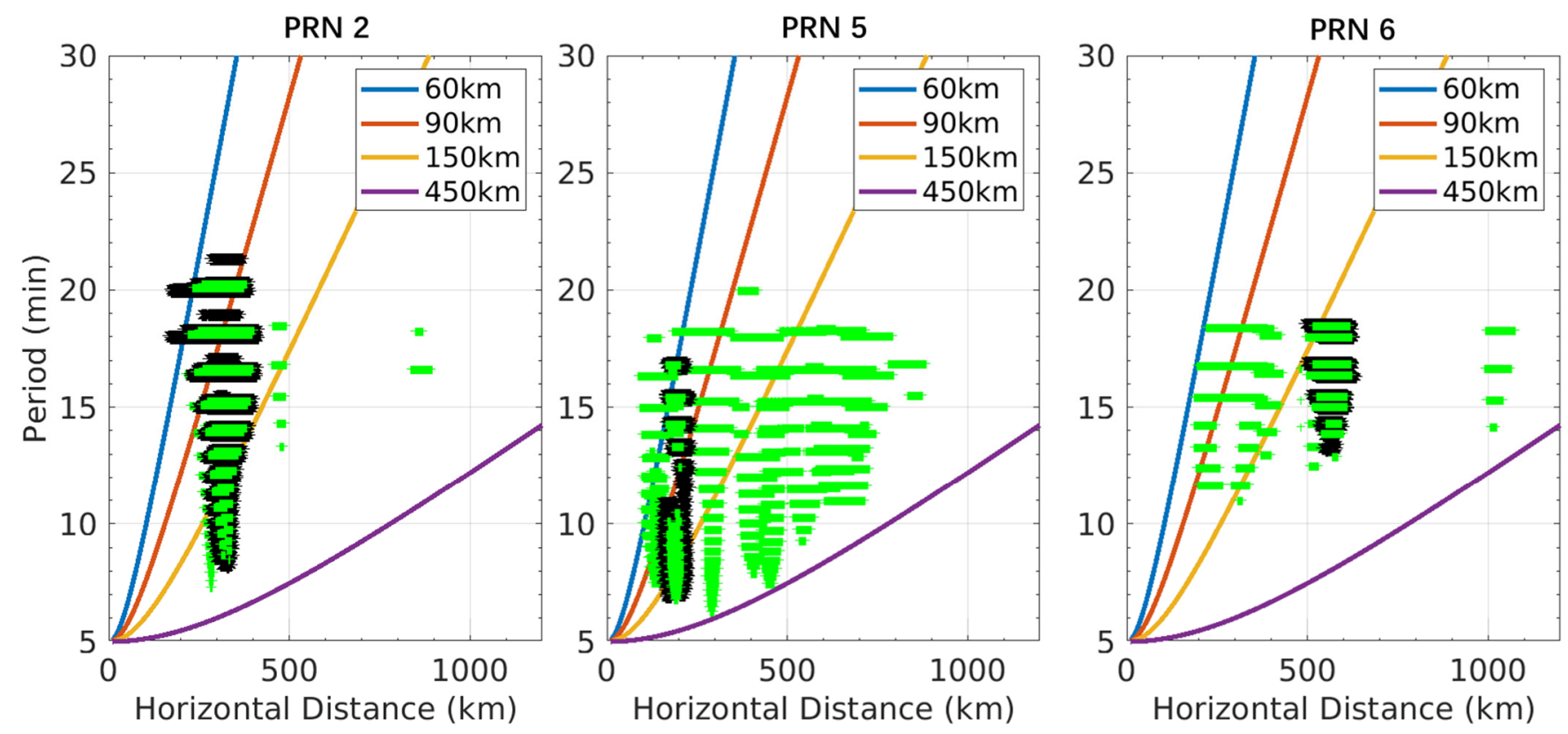

Figure 8. Horizontal propagation distance versus GW period: comparison of theoretical and measured values. The scatters represent the presence of GW, i.e., observations with energy greater than a certain threshold after the S-transformation. The black scatters show are observations from stations in Hongkong. The green scatters represent other stations. Four curves of different colors show the theoretical relationship between the period of the observable GW and the horizontal propagation distance at different heights in the ionosphere.

The three subplots in Figure 8 represent the observations of PRN 2, 5, and 6, respectively. From the observations at the Hong Kong station indicated by the black scattered points in Figure 8, it can be seen that the disturbance observed at PRN 5 is closest to the center of the thunderstorm, PRN 2 is the next closest, and PRN 6 is the farthest. This is consistent with that shown in Figure 4.

In the left panel, most of the measured horizontal propagation distances of GWs lie between 250 and $500 \mathrm{~km}$, between the blue and yellow lines. This reveals that the observed GWs are mainly present at the $\mathrm{D}, \mathrm{E}$, and bottom of the $\mathrm{F}$ layers.

In comparison with PRN 2 and PRN 6, PRN 5 observes this disturbance from a wider length range. In the range of horizontal propagation distance less than $500 \mathrm{~km}$, the disturbances have periods of 6-20 min. When the distance is greater than $500 \mathrm{~km}$, only disturbances with a period of $10 \mathrm{~min}$ or more are observed. The disturbances are present in almost the entire region from the D to F layers. The purple line of the PRN5 subplot of Figure 8, enveloping the measured values, indicates the variation of the shortest period with horizontal distance. The envelope line is located between $150 \mathrm{~km}$ and $450 \mathrm{~km}$ of ionospheric altitude. By the black and green scatters and the four solid lines, it can be inferred that the higher the region where the ionospheric GWs exist, the longer the period. The GNSS measurements show that there are longer periods of GWs with the increase of propagation distance, which is consistent with the propagation theory of GW.

\subsection{Properties of the Propagation Velocity of Perturbation}

When GWs propagate into the ionosphere, the measured TECU exhibits clear wavelike structures, as shown in Figure 9. The peaks and troughs of the disturbed region are imaged on the IPP trajectory at different moments and different propagation distances, making it possible to estimate the propagation speeds of the GWs. 


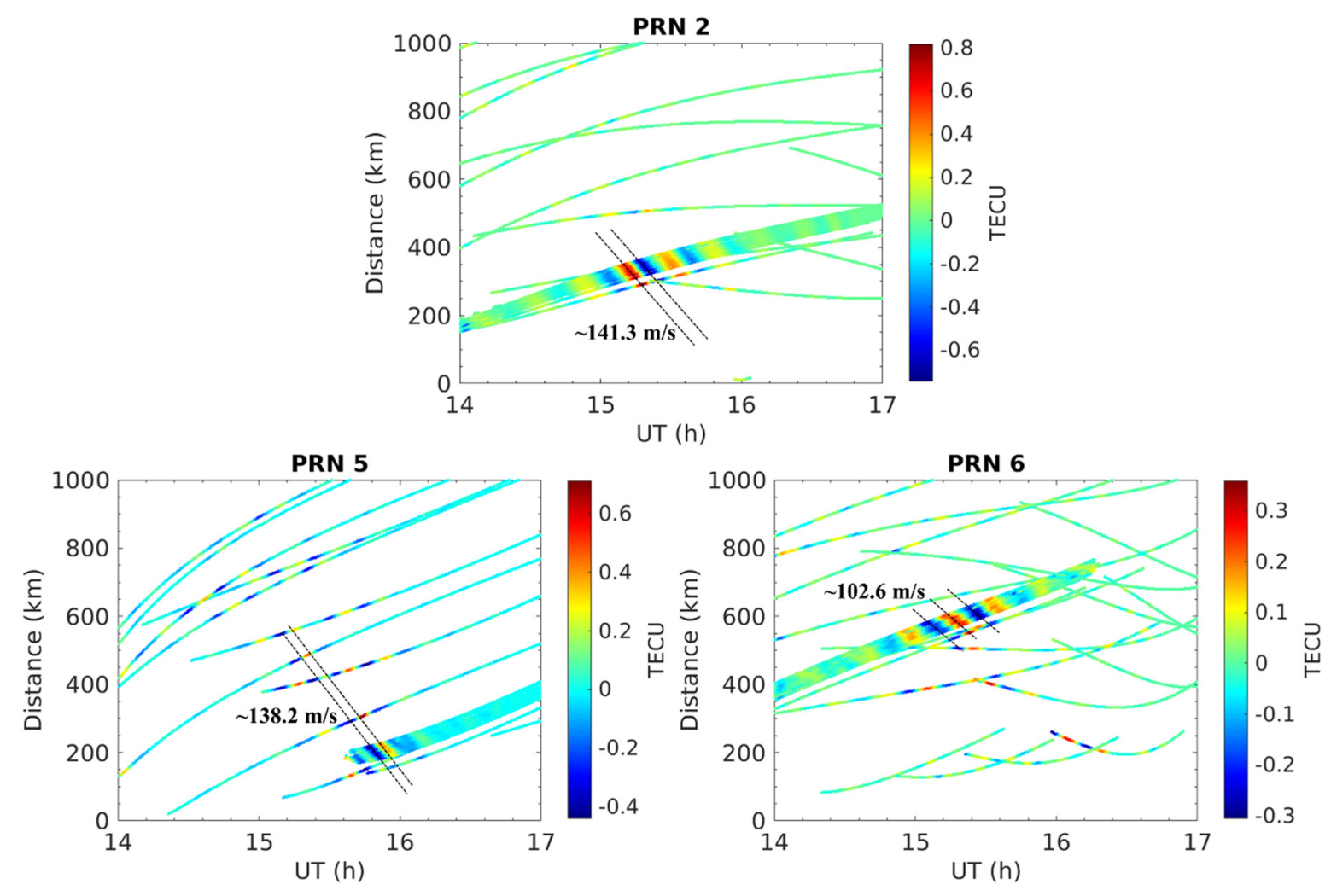

Figure 9. Determination of the propagation velocity of GWs. The horizontal coordinate indicates the UTC, the vertical coordinate indicates the distance of the IPP from the center of the thunderstorm, and the colorbar indicates the magnitude of the ionospheric disturbance.

In Figure 9, the wave peaks or troughs on the IPP at different stations are fitted linearly. The slope of the fitted line is the propagation speed of the GWs [15,17]. For instance, $141.3 \mathrm{~m} / \mathrm{s}$ is measured by PRN 2, $138.2 \mathrm{~m} / \mathrm{s}$ by PRN 5, and $102.6 \mathrm{~m} / \mathrm{s}$ by PRN 6, as shown in Figure 9. The disturbance observed by PRN 2 is during 15:00-16:00 and propagates at a distance of 200-400 km. During the same period, the disturbances observed by PRN 6 are located at a propagation distance of $600 \mathrm{~km}$, which means that the IPP of PRN 6 is further from the source of the thunderstorms at this time slot. This indicates more than one type of GWs generated by the thunderstorms and the different IPP distances observing the waves with different wave propagation speeds.

The observation of PRN 5 is located close to 16:00, around $200 \mathrm{~km}$. Although the measured wave speeds of PRN 2 and 5 are close, they are observed at different times and places. PRN 2 firstly observes a disturbance with a speed of $\sim 140 \mathrm{~m} / \mathrm{s}$ at a further location before PRN 5 captures a disturbance with a slower speed at a closer location. This implies that the satellites are not observing the same GWs but successively excited GWs. In conjunction with Figure 2, it can be shown that both the thunderstorm and its generated GWs possess temporal continuity.

\section{Discussion}

\subsection{Extraction Methods of Gravity Waves and the Observability at Low Latitudes}

The GWs perturbations triggered by thunderstorms in the GNSS signals at lowlatitude regions are difficult to be extracted separately. However, the present study successfully demonstrates that GWs perturbations at low latitudes can be retrieved and analyzed by using appropriate methods.

Firstly, for the accurate calculation of ionospheric perturbations, the accuracy of using only code observations is insufficient. If only carrier smoothing pseudorange is used, the hidden cycle slips or gross errors of the carrier phase may be difficult to detect, leading 
to an abrupt change in the TEC calculation. This abrupt change could bring in otherwise non-existent periodic terms through Hatch filtering. To avoid this issue, the detection of cycle slips using only the time difference of the carrier can be utilized to calculate the ionospheric perturbations with relatively high accuracy $[17,18]$.

Secondly, polynomial fitting can remove the long-period trend, and it is a necessary step to extract short-period ionospheric perturbations [25]. At mid-latitudes, this approach is effective [6,7]. However, at low latitudes, due to the complex ionospheric variations, the disturbances of TEC obtained after detrending only by polynomial fitting still contain multiple periods. Therefore, the employment of band-pass filters is necessary to extract the perturbations for a known range of periods $[8,10,15]$. Studies $[6,26]$ have shown that GWs can be observed at night with the aid of bandpass filters, both at mid and low latitudes. Statistical studies have proved the validity of such methods $[9,10]$.

If the geomagnetic field is active on that day, the mixed perturbations may be introduced into the TEC, causing inaccurate GWs extraction. The Dst index shows that the range of the geomagnetic is -33 to $5 \mathrm{nT}$ and the Kp index is lower than 5 from March 29 to April 5, 2019, both indicating that the geomagnetic is quiet. Therefore, the TEC results of this study can exclude the effects of large-scale geomagnetic disturbances.

A detailed analysis that GWs at $\sim 114^{\circ} \mathrm{E}, 24^{\circ} \mathrm{N}$ are indeed triggered by thunderstorms is given in this paper. Besides, in Figure 3, disturbances with a period of $20 \mathrm{~min}$ are present over Vietnam and Laos $\left(\sim 105^{\circ} \mathrm{E}, 18^{\circ} \mathrm{N}\right)$. There is no evidence that the disturbances are associated with thunderstorms. Similar disturbances propagating in the southwest-northeast direction are also observed at low latitudes in China [27]; meanwhile, the southwestoriented Traveling Ionospheric Disturbances (TIDs) are observed in India [28]. There are possible causes for this kind of TID: (1) the electric field excites the Electrical Medium-Scale TID (EMSTID) propagating southwestward in the northern hemisphere [29]; and (2), these disturbances are caused by the perturbation of the neutral winds [30].

\subsection{Periodic Characteristics and Perturbation Magnitudes}

Some studies have shown that the GWs driven by thunderstorms are less than 20 min, and the periods of GWs triggered from the troposphere are about $20 \mathrm{~min}$ [31]. High frequency (period $4 \mathrm{~min}$ ) shocks exhibits when a thunderstorm passes [7]. The disturbances with infrasound periods of 1-5 min could be found during thunderstorms [8]. Besides, some studies suggested that the period should be $15 \mathrm{~min}$ to $1 \mathrm{~h} \mathrm{[32].} \mathrm{At} 150 \mathrm{~km}$ high, there are GWs of $0.2-1 \mathrm{mHz}(16.7-83.3 \mathrm{~min})$ with $0.4-1.5 T E C U$. At 250 and $375 \mathrm{~km}$, there are secondary GWs of $0.2-0.5 \mathrm{mHz}$ (33.3-83.3 $\mathrm{min}$ ) with 0.2-0.6 TECU [33]. GWs of 30-100 min have been shown for low-latitude thunderstorms in China [11], while the perturbation period is longer than one hour in West Africa [12].

In our study, the right panel of Figures 2 and 3 show that waves with periods of 6-20 min exist in the presence of thunderstorms. Further, the propagation theory of GWs in the atmosphere is introduced to confirm that the waves are very likely triggered by those thunderstorms within a certain distance.

Notably, the left panel of Figures 2 and 3 shows that longer period disturbances are present both on thunderstorm days and non-thunderstorm days. This suggests that some long-period disturbances are not dependent on thunderstorms.

For the magnitude of GWs, Erin et al. [6,9] and Rahmani et al. [34] use 0.08 TECU as the threshold, which is also used in this paper for the 6-16 min perturbation. In Figures 5 and 9, PRN 6 has the smallest perturbation magnitude; however, it reaches 0.3 TECU, which exceeds the threshold of 0.08 TECU [9] and can therefore be judged as a GW perturbation.

For the periods of the perturbations longer than $20 \mathrm{~min}$, the magnitudes of perturbation are 0.5-1.5 TECU during the day in West Africa [12] and even up to 10 TECU or 100 TECU in low latitude China [11] during the night. The presence of inherent longperiod perturbations at low latitudes may be caused by the PRE during sunset [35] or a nighttime plasma bubble due to upward currents [36]. Numerous studies have shown that the magnitude of perturbations propagating from the atmosphere to the ionosphere is 
quite low $[13-15,17,37,38]$. Therefore, in our study, the extreme perturbations of TECUs are treated as anomalies.

Large magnitudes of perturbations found during the thunderstorms are highly likely caused by the cycle slips in GNSS receivers when the ionosphere is disturbed. The measured TEC in this scenario may have large biases due to lightning [11]. However, the ionosphere at low latitudes is unstable, and the presence of thunderstorms or strong convective weather may exacerbate this instability, resulting in larger magnitudes of longperiod perturbations $[9,10,39]$. Both of these scenarios can produce larger GNSS signal perturbations, which are not directly caused by GWs from thunderstorms.

\subsection{Properties of the Propagation Speed}

The theory and results of Vadas and Liu [22] show that most of the GWs that originated from the bottom atmosphere have propagation speeds of less than 250-300 m/s. Studies of the typhoon-induced TID show the wave propagation velocity from 118-186 m/s [15] and around $240 \mathrm{~m} / \mathrm{s}$ [13]. Song et al. [38] give wave velocities that are $143 \mathrm{~m} / \mathrm{s}$ and $268 \mathrm{~m} / \mathrm{s}$ for two types of TID during a typhoon. In our cases, we show that the wave propagation speeds during thunderstorms are from $102 \mathrm{~m} / \mathrm{s}$ to $141 \mathrm{~m} / \mathrm{s}$. Extreme weathers like typhoons and thunderstorms have in common that the characteristics of the dynamics time scales are comparable. Therefore, the wave triggered by the extreme weathers may have similar propagation velocities, i.e., both less than 250-300 m/s. However, propagation velocities larger than $150 \mathrm{~m} / \mathrm{s}$ were not found in this study. This is most likely because the waves with propagation velocities larger than $150 \mathrm{~m} / \mathrm{s}$ may exist but are not captured due to the sparse deployment stations. BeiDou's geostationary orbit (GEO) satellite may provide a solution to this problem $[40,41]$.

\section{Conclusions}

This study shows an effective method to exact small ionospheric perturbations from GNSS signals and presents clear cases that the GWs induced by thunderstorms can be observed from low latitudes by GNSS. The characteristics of the GWs triggered by lowlatitude thunderstorms in terms of wave magnitudes, periods, and proparation speed are provided. The main conclusions are as follows:

(1) Ionospheric GW structures in low latitudes China are detectable by GNSS by proper methods, and the characteristics of the ionospheric GWs can be extracted from the complex variations of the low-latitude ionosphere.

(2) Tropospheric thunderstorms can drive ionospheric perturbations with magnitudes of $0.2-0.8$ TECU and periods of 6-20 min. The disturbance is temporally located $1-2 \mathrm{~h}$ after the peak of the thunderstorm and spatially located $200-800 \mathrm{~km}$ from the center of the thunderstorm. The propagation velocity is less than $250-300 \mathrm{~m} / \mathrm{s}$, indicating that the observed GWs are propagated from the bottom atmosphere to the ionosphere.

(3) The theory of GW period variation with propagation distance in the atmosphere is introduced. The variation of the theoretical period with propagation distance of the model is consistent with the observed period, indicating that GWs triggered by the thunderstorms have impacts on the distant ionosphere from the thunderstorms, not directly above the thunderstorms.

This analytical framework can be equally applied to the analysis of GWs triggered by thunderstorms or strong convection in other regions. In addition, this study illustrates that GWs can be observed both during the day and at night. For sustained thunderstorms, GWs can be continuously activated, leading to different periodic perturbations of the ionosphere at different altitudes. The presence of GWs both with and without thunderstorms should be caused by other physical processes.

In this paper, ionospheric acoustic waves (disturbances of less than 6 min period) and disturbances larger than $20 \mathrm{~min}$ are not included. At low latitudes, the correlation between such disturbances and thunderstorms and the validation of physical models are still to be further investigated. 
Author Contributions: Conceptualization, Z.Y.; methodology, Z.Y. and T.L.; software, Z.Y. and T.L.; investigation, T.L.; data curation, Z.D. and W.N.; writing-original draft preparation, T.L.; writing-review and editing, Z.Y., Z.D. and W.N.; supervision, Z.Y. and G.X.; funding acquisition, Z.Y. and G.X. All authors have read and agreed to the published version of the manuscript.

Funding: This research is funded by the Key-Area Research and Development Program of Guangdong Province, Grant No. 2020B0303020001; the Shenzhen Science and Technology Program, Grant No. KQTD20180410161218820; and the National Key Laboratory of Electromagnetic Environment, Grant No.202001004.

Institutional Review Board Statement: Not applicable.

Informed Consent Statement: Not applicable.

Data Availability Statement: The raw GNSS data in RINEX format is from https:/ / data.earthquake. cn/ (accessed on 12 October 2020) and https:/ / www.geodetic.gov.hk/ (accessed on 5 April 2021); The lightning data of FY-4 satellite is from http:/ / www.nsmc.org.cn/en/NSMC/Home/index.html (accessed on 16 July 2021); The Dst index is available here: http:/ /wdc.kugi.kyoto-u.ac.jp/dstae/ index.html (accessed on 1 June 2021).

Acknowledgments: Acknowledgement for the data support from "China Earthquake Networks Center, National Earthquake Data Center (http: / / data.earthquake.cn (accessed on 12 October 2020))", "Survey and Mapping Office Lands Department (http:/ / www.geodetic.gov.hk/sc/gi/gsi_index.htm (accessed on 5 April 2021))", “National Data Center for Meteorological Sciences (http:/ / data.cma.cn/ (accessed on 16 July 2021))", and "Institute of Electrical Engineering Chinese Academy of Sciences ( http:/ /www.iee.ac.cn/ (accessed on 13 April 2021))". Acknowledgement for the data support from http:/ / wdc.kugi.kyoto-u.ac.jp/dstae/index.html (accessed on 1 June 2021).

Conflicts of Interest: The authors declare no conflict of interest.

\section{References}

1. Yang, L.; Fu, L.; Wang, J.; Zhang, C. Studying Ionosphere Responses to a Geomagnetic Storm in June 2015 with Multi-Constellation Observations. Remote Sens. 2018, 10, 666.

2. Yasyukevich, Y.; Vasilyev, R.; Ratovsky, K.; Setov, A.; Globa, M.; Syrovatskii, S.; Yasyukevich, A.; Kiselev, A.; Vesnin, A. Small-Scale Ionospheric Irregularities of Auroral Origin at Mid-latitudes during the 22 June 2015 Magnetic Storm and Their Effect on GPS Positioning. Remote Sens. 2020, 12, 1579. [CrossRef]

3. Vadas, S.L.; Azeem, I. Concentric Secondary Gravity Waves in the Thermosphere and Ionosphere Over the Continental United States on March 25-26, 2015 From Deep Convection. J. Geophys. Res. Space Phys. 2021, 126, 028275. [CrossRef]

4. Zhai, C.; Yao, Y.; Kong, J. Three-dimensional reconstruction of seismo-traveling ionospheric disturbances after March 11, 2011, Japan Tohoku earthquake. J. Geod. 2021, 95, 1-12. [CrossRef]

5. Freeshah, M.; Zhang, X.; Şentürk, E.; Adil, M.; Mousa, B.; Tariq, A.; Ren, X.; Refaat, M. Analysis of Atmospheric and Ionospheric Variations Due to Impacts of Super Typhoon Mangkhut (1822) in the Northwest Pacific Ocean. Remote Sens. 2021, $13,661$. [CrossRef]

6. Erin, L.; Shao, X.M.; Kendrick, A.K.; Carrano, C.S. Ionospheric acoustic and gravity waves associated with midlatitude thunderstorms. J. Geophys. Res. Space Phys. 2015, 120, 6010-6020.

7. Lay, E.H.; Shao, X.-M.; Carrano, C.S. Variation in total electron content above large thunderstorms. Geophys. Res. Lett. 2013, 40, 1945-1949. [CrossRef]

8. Shao, X.-M.; Lay, E.H. The origin of infrasonic ionosphere oscillations over tropospheric thunderstorms. J. Geophys. Res. Space Phys. 2016, 121, 6783-6798. [CrossRef]

9. Erin, H.L. Ionospheric Irregularities and Acoustic/Gravity Wave Activity Above Low-Latitude Thunderstorms. Geophys. Res. Lett. 2018, 45, 90-97.

10. Tang, L.; Chen, W.; Louis, O.-P. Statistical Observation of Thunderstorm-Induced Ionospheric Gravity Waves above Low-Latitude Areas in the Northern Hemisphere. Remote Sens. 2019, 11, 2732. [CrossRef]

11. Kumar, S.; Chen, W.; Liu, Z.; Singh, R.P. Thunderstorm-/lightning-induced ionospheric perturbation: An observation from equatorial and low-latitude stations around Hong Kong. J. Geophys. Res. Space Phys. 2017, 122, 9032-9044. [CrossRef]

12. Ogunsua, B.O.; Srivastava, A.; Bian, J.; Qie, X.; Wang, D.; Jiang, R.; Yang, J. Significant Day-time Ionospheric Perturbation by Thunderstorms along the West African and Congo Sector of Equatorial Region. Sci. Rep. 2020, 10, 8466. [CrossRef]

13. Jian, K.; Yao, Y.; Xu, Y.; Kuo, C.; Zhang, L.; Liu, L.; Zhai, C. A clear link connecting the troposphere and ionosphere: Ionospheric reponses to the 2015 Typhoon Dujuan. J. Geod. 2017, 91, 1087-1097.

14. Chen, J.; Zhang, X.; Ren, X.; Zhang, J.; Freeshah, M.; Zhao, Z. Ionospheric disturbances detected during a typhoon based on GNSS phase observations: A case study for typhoon Mangkhut over Hong Kong. Adv. Space Res. 2020, 66, 1743-1753. [CrossRef] 
15. Wen, Y.; Jin, S. Traveling Ionospheric Disturbances Characteristics during the 2018 Typhoon Maria from GPS Observations. Remote Sens. 2020, 12, 746. [CrossRef]

16. Wen, Y.; Zhang, Q.; Xu, J.; Li, Q.; Gao, H. Propagation characteristics of mesospheric concentric gravity waves excited by a thunderstorm. Chin. J. Geophys. 2019, 62, 1218-1229.

17. Savastano, G.; Komjathy, A.; Verkhoglyadova, O.; Mazzoni, A.; Crespi, M.; Wei, Y.; Mannucci, A.J. Real-Time Detection of Tsunami Ionospheric Disturbances with a Stand-Alone GNSS Receiver: A Preliminary Feasibility Demonstration. Sci. Rep. 2017, 7, 46607. [CrossRef]

18. Cai, C.; Liu, Z.; Xia, P.; Dai, W. Cycle slip detection and repair for undifferenced GPS observations under high ionospheric activity. GPS Solut. 2012, 17, 247-260. [CrossRef]

19. Haaser, R.; Lay, E.H.; Junor, W. Analysis framework for systematically studying ionospheric response to impulsive events from below. Radio Sci. 2017, 52, 1149-1169. [CrossRef]

20. Hines, C.O. Internal atmospheric gravity waves at ionospheric heights. Can. J. Phys. 1960, 38, 1424-1427. [CrossRef]

21. Fritts, D.C.; Alexander, M.J. Correction to "Gravity wave dynamics and effects in the middle atmosphere". Rev. Geophys. 2012, 50, RG3004. [CrossRef]

22. Vadas, S.L.; Liu, H.-L. Generation of large-scale gravity waves and neutral winds in the thermosphere from the dissipation of convectively generated gravity waves. J. Geophys. Res. Space Phys. 2009, 114, A10310. [CrossRef]

23. Nayak, C.; Yiit, E. GPS-TEC Observation of Gravity Waves Generated in the Ionosphere During 21 August 2017 Total Solar Eclipse. J. Geophys. Res. Space Phys. 2018, 123, 725-738. [CrossRef]

24. Stockwell, R.G.; Mansinha, L.; Lowe, R.P. Localization of the complex spectrum: The S transform. IEEE Trans. Signal Process. 1996, 44, 998-1001. [CrossRef]

25. Freeshah, M.A.A.; Zhang, X.; Chen, J.; Zhao, Z.; Osama, N.; Sadek, M.; Twumasi, N. Detecting Ionospheric TEC Disturbances by Three Methods of Detrending through Dense CORS During A Strong Thunderstorm. Ann. Geophys. 2020, 63, 667. [CrossRef]

26. Yang, H.; Moreno, E.M.; Hernández-Pajares, M. ADDTID: An Alternative Tool for Studying Earthquake/Tsunami Signatures in the Ionosphere. Case of the 2011 Tohoku Earthquake. Remote Sens. 2019, 11, 1894. [CrossRef]

27. Wu, K.; Xu, J.; Wang, W.; Sun, L.; Yuan, W. Interaction of Oppositely Traveling Medium-scale Traveling Ionospheric Disturbances Observed in Low Latitudes During Geomagnetically Quiet Nighttime. J. Geophys. Res. Space Phys. 2020, 126, e2020JA028723.

28. Bhat, A.H.; Ganaie, B.A.; Ramkumar, T.K.; Chaitanya, P.P.; Malik, M.A. Simultaneous detection of medium-scale traveling ionospheric disturbances and ionospheric plasma irregularities over Srinagar, J\&K, India. Acta Geophys. 2021, 69, 1-15. [CrossRef]

29. Narayanan, V.L.; Patra, A.K.; Gurubaran, S.; Chaitanya, P.P.; Emperumal, K. Coincident Airglow, VHF Radar, and Ionosonde Observations of Electrified Medium-Scale Traveling Ionospheric Disturbances in the Equatorial Latitudes. Geophys. Res. Lett. 2019, 46, 7173-7181. [CrossRef]

30. Jonah, O.F.; Zhang, S.; Coster, A.J.; Goncharenko, L.P.; Erickson, P.J.; Rideout, W.; de Paula, E.R.; de Jesus, R. Understanding Inter-Hemispheric Traveling Ionospheric Disturbances and Their Mechanisms. Remote Sens. 2020, 12, 228. [CrossRef]

31. Vadas, S.L.; Nicolls, M.J. Temporal evolution of neutral, thermospheric winds and plasma response using PFISR measurements of gravity waves. J. Atmos. Solar-Terr. Phys. 2009, 71, 744-770. [CrossRef]

32. Oinats, A.V.; Nishitani, N.; Ponomarenko, P.; Berngardt, O.; Ratovsky, K.G. Statistical characteristics of medium-scale traveling ionospheric disturbances revealed from the Hokkaido East and Ekaterinburg HF radar data. Earth Planets Space 2016, 68, 8 [CrossRef]

33. Chou, C.-C.; Dai, J.; Kuo, C.-L.; Huang, T.-Y. Simultaneous observations of storm-generated sprite and gravity wave over Bangladesh. J. Geophys. Res. Space Phys. 2016, 121, 9222-9233. [CrossRef]

34. Rahmani, Y.; Alizadeh, M.M.; Schuh, H.; Wickert, J.; Tsai, L.-C. Probing vertical coupling effects of thunderstorms on lower ionosphere using GNSS data. Adv. Space Res. 2020, 66, 1967-1976. [CrossRef]

35. Abdu, M.A.; Pancheva, D. Aeronomy of the Earth's Atmosphere and Ionosphere; IAGA Special Sopron Book Series; Abdu, M.A., Pancheva, D., Bhattacharyya, A., Eds.; Springer: Berlin/Heidelberg, Germany, 2011.

36. Kuo, C.L.; Lee, L.C. Ionospheric plasma dynamics and instability caused by upward currents above thunderstorms. J. Geophys. Res. Space Phys. 2015, 120, 3240-3253. [CrossRef]

37. Vadas, S.L.; Liu, H.-L. Numerical modeling of the large-scale neutral and plasma responses to the body forces created by the dissipation of gravity waves from $6 \mathrm{~h}$ of deep convection in Brazil. J. Geophys. Res. Space Phys. 2013, 118, 2593-2617. [CrossRef]

38. Song, Q.; Ding, F.; Zhang, X.; Liu, H.; Mao, T.; Zhao, X.; Wang, Y. Medium-Scale Traveling Ionospheric Disturbances Induced by Typhoon Chan-hom Over China. J. Geophys. Res. Space Phys. 2019, 124, 2223-2237. [CrossRef]

39. Erin, L.; Parker, P.A.; Light, M.; Carrano, C.S.; Debchoudhury, S.; Haaser, R.A. Midlatitude Ionospheric Irregularity Spectral Density as Determined by Ground-Based GPS Receiver Networks. J. Geophys. Res. Space Phys. 2018, $123,5055-5067$.

40. Padokhin, A.; Tereshin, N.; Yasyukevich, Y.; Andreeva, E.; Nazarenko, M.; Yasyukevich, A.; Kozlovtseva, E.; Kurbatov, G. Application of BDS-GEO for studying TEC variability in equatorial ionosphere on different time scales. Adv. Space Res. 2018, 63, 257-269. [CrossRef]

41. Huang, F.; Lei, J.; Otsuka, Y.; Luan, X.; Liu, Y.; Zhong, J.; Dou, X. Characteristics of Medium-Scale Traveling Ionospheric Disturbances and Ionospheric Irregularities at Mid-Latitudes Revealed by the Total Electron Content Associated With the Beidou Geostationary Satellite. IEEE Trans. Geosci. Remote Sens. 2020, 59, 1-7. [CrossRef] 